(C) ACM, 2008. This is the author's version of the work. It is posted here by permission of ACM for your personal use. Not for redistribution. The definitive version was published in MobileHCl '08: Proceedings of the 10th international conference on Human computer interaction with mobile devices and services, http://doi.acm.org/10.1145/1409240.1409325

\title{
Understanding Mobile Information Needs
}

\author{
Karen Church \\ Adaptive Information Cluster \\ University College Dublin \\ Belfield, Dublin 4, Ireland \\ karen.church@ucd.ie
}

\author{
Barry Smyth \\ Adaptive Information Cluster \\ University College Dublin \\ Belfield, Dublin 4, Ireland \\ barry.smyth@ucd.ie
}

\begin{abstract}
In this paper we describe the results of a four-week diary study of mobile information needs, focusing on the intent behind mobile needs and the importance on context on such needs. We identified three key intents among diary entries: informational, geographical and personal information management (PIM). Furthermore, we found many of these information needs have high temporal and location dependencies.
\end{abstract}

\section{Categories and Subject Descriptors}

H.m [Information Systems]: Miscellaneous

\section{General Terms}

Experimentation, Human factors

\section{Keywords}

Diary Study, Mobile, Information Needs, Intent

\section{INTRODUCTION}

The mobile world has experienced phenomenal growth in recent times. In 2007, the number of mobile subscribers reached 3.5 billion worldwide ${ }^{1}$. Up until recently operator portals have dominated mobile access and recent reports highlight the growing popularity of off-carrier-portal content $^{2}$. Therefore, it is likely that search will become an increasingly popular way to locate content on the Mobile Internet, especially as off-portal content grows.

There have been a number of studies to date that examine mobile search behaviour. The most recent analysis of Google's mobile search [3] shows that mobile queries are short (2.6 terms), adult-content is prevalent and mobile queries are becoming less homogenous [3]. Recent work by Church et al. [2] highlighted the dominance of browsing as

\footnotetext{
${ }^{1}$ http://www.flashdevices.net/2007/11/35-billion-mobilephone-subscribers.html

${ }^{2}$ http://www.mmaglobal.com/offportal.pdf
}

Permission to make digital or hard copies of all or part of this work for personal or classroom use is granted without fee provided that copies are not made or distributed for profit or commercial advantage and that copies bear this notice and the full citation on the first page. To copy otherwise, or republish, to post on servers or to redistribute to lists, requires prior specific permission and/or a fee.

MobileHCI September 2-5, 2008, Amsterdam, the Netherlands.

Copyright is held by the author/owner(s). an information access mode on the Mobile Internet (94\% of sessions). They also found that mobile queries tend to be short and vague (2.1 terms), with adult-content representing $50 \%$ of the top 500 queries analysed. These studies are important because they provide insights into what mobile users look for and how they search for information online.

Mobile search differs significantly from Web search, not just because of mobile devices but also because peoples information needs differ when mobile. In order to offer effective mobile services we need to understand mobile users in more detail. For this to happen, however, we need to better understand the characteristics of mobile information needs. So as a complimentary study to previous mobile analyses $[2$, $3]$, we have carried out a diary study of mobile information needs. The goal of our work is to understand the intent behind mobile information needs and how those information needs changes based on context.

\section{THE DIARY STUDY}

Here we describe the results of a recent diary study to evaluate mobile information needs. The method has recently been used in a number of mobile studies, e.g. [4].

\subsection{Participants \& Procedure}

The 20 participants (13 females and 9 males) ranged in age between 20 and 60 , with a mean age of 31 (std. dev. 10.75). All of our participants own a mobile phone and have experience with standard phone facilities. The study ran for a period of 4 weeks, from the $5^{\text {th }}$ Nov $2007-3^{\text {rd }}$ Dec 2007. Although we are primarily interested in mobile needs we allowed users to log all information needs in order to investigate whether key differences existed between information needs arising when mobile compared to those that occur while at home or at work. We define mobile as being away from their own home or away from their desk at work.

Participants were asked to log the date and time, their current location and the information need in a small notebook. Participants were also encouraged to include any comments they thought might be relevant (although this was optional). If participants were unable to note an information need or if they forgot to note an information need at a specific time, they were advised to do so later in the day. We wanted the study to capture information needs as they occurred but in the most natural way possible. For this reason we did not want to inundate users with daily/hourly reminders about the study. Instead we sent a weekly reminder to participants via SMS. No other incentives were provided to participants. We conducted an initial survey of participants, gathering 
data on their mobile phone experience and preference information. We also asked participants for clarification of any unclear entries at the end of the study.

\subsection{Summary Results}

Our study generated 405 diary entries, with an average of 20.3 entries per person $(\min =6, \max =43$, st. dev. $=11.2)$. Although we allowed participants to log their information needs in a variety of location contexts, we found that the majority of the entries $(>67 \%)$ where generated when users were mobile. That is, away from their desk, commuting, traveling abroad or on-the-go. Probably the most striking result is that the most popular context, by a significant lead, is on-the-go with $>34 \%$ of entries, suggesting that many users have a variety of information needs when they are away from their familiar contexts.

Previously, Broder [1] classified Web queries according to 3 classes of intent: (1) Navigational queries refer to a class of queries where the immediate intent is to reach a particular site. (2) Informational queries are those that involve the user attempting to find information online - that is, the user is probably interested in reading this information online but no further interaction is expected. Finally, (3) transactional queries refer to queries in which the purpose is to visit a site where additional interactions will occur. For example, shopping, downloading files, etc. In general, all adult-related queries are classified as transactional.

We found a very different distribution of intent among our diary entries, suggesting the need for the two new categories to reflect the natural biases that exist within the mobile space (See Table 1).

\begin{tabular}{|l|l|}
\hline Goal & \% Entries \\
\hline 1. Informational & 58.3 \\
\hline 2. Geographical & 31.1 \\
2.1 Local Explicit & 12.8 \\
2.2 Local Implicit & 14.8 \\
2.3 Directions & 3.5 \\
\hline 3. Personal Information Management & 10.6 \\
\hline
\end{tabular}

Table 1: Results of classifying entries by intent

Informational needs are all focused on the goal of obtaining information about a topic, e.g. "Is the Aresnal game on TV tonight?". Geographical needs are focused on the goal of finding an answer to a question, however, the information need and the answer expected is dependent on location in some form. We define 3 subclasses of this category: local explicit, local implicit and directions. Local explicit describes an information need in which the users current location has a definite impact on the information need and the answer expected. These needs are identified by the presence of physical location/proximity keywords, e.g., "Nearest Pennies or Dunnes Stores". Local implicit describes needs in which the user is searching for a physical location either directly or indirectly. Most of these needs are identified by the question where, e.g., "Where do I get coffee?". Finally directions, describes an information need in which the users is looking for directions or an appropriate route to a physical location, e.g., "Direction from Belfast to Randaldstown". Personal information management (PIM) needs are focused on the goal of finding out something private relating to the individual, i.e. personal to the user in question. Normally these are questions that only the individual can answer or to answer the question, input from the individual is required. PIM needs encompass personal items, friend requests, scheduling tasks and to-do list items, e.g., "What are my airline loyalty pin codes?", etc.

The results (Table 1) show that almost $42 \%$ of the diary entries are non-informational and $>30 \%$ of entries are geographical in nature, suggesting a high dependency on location in mobile needs. Looking closer at the geographical category we find local explicit entries to be popular; $12.8 \%$ of all entries (41.3\% of all geographical entries). Thus endusers often explicitly indicate that their current location is of definite importance to the information need and the answer they would like to receive. PIM needs represent almost $11 \%$ of the diary entries. These entries correspond to needs that are personal to the end-user. However, many of these entries cannot be answered easily by current mobile devices, e.g, "What are my airline loyalty pin codes?", thus highlighting the need for new PIM services on mobile handsets to help deal with these types of scenarios.

\section{CONCLUSIONS}

One important finding that emerged from our study is the importance of context, in particular location and time on information needs, especially when the user is on-the-go. We found that over $30 \%$ of information needs were geographical in nature, i.e. location dependent. Most participants sought after services/products within close vicinity to where they live, work or interact and many users included explicit location cues in their entries, e.g, nearest $X$. We also found a number of diary entries expressing information needs with temporal dependencies. For example, $8.4 \%$ of entries included explicit temporal cues, "Is there anything good on tv tonight?". Furthermore, most of the geographical information needs were temporally dependent, even though most did not include explicit temporal cues.

These results suggest that it is time for a radical rethinking of mobile search. Current approaches to mobile search are limited by the traditional query-based search interface on which they are based, one in which context and preferences play an ad-hoc role in guiding search. In order to offer mobile users an improved mobile search experience, we need to develop new types of context-sensitive mobile services that take full advantage of temporal, geographic, and preference-based contexts of mobile subscribers.

\section{ACKNOWLEDGEMENTS}

This material is based on works supported by the Science Foundation Ireland under Grant No. 03/IN.3/I361.

\section{REFERENCES}

[1] A. Broder. A taxonomy of web search. SIGIR Forum, 36(2):3-10, 2002.

[2] K. Church, B. Smyth, P. Cotter, and K. Bradley. Mobile information access: A study of emerging search behavior on the mobile internet. ACM Transactions on the Web, 1(1):4, 2007.

[3] M. Kamvar and S. Baluja. Deciphering trends in mobile search. Computer, 40(8):58-62, 2007.

[4] T. Sohn, K. A. Li, W. G. Griswold, and J. D. Hollan. A diary study of mobile information needs. In Proceedings of the CHI'08, pages 433-442. ACM, 2008. 